\title{
Molecular epidemiology of respiratory syncytial virus among children and adults in India 2016 to 2018
}

\author{
Suresh S. Bandla ${ }^{1} \cdot$ Santhosha Devadiga ${ }^{1} \cdot$ Rushil Bhatt $^{1} \cdot$ Oliver C. Dsa ${ }^{1} \cdot$ Arunkumar Govindakarnavar $^{1,2}(\mathbb{D}$
}

Received: 12 June 2020 / Accepted: 25 June 2021 / Published online: 15 September 2021

(c) The Author(s) 2021

\begin{abstract}
Respiratory syncytial virus (RSV) is a common cause of respiratory tract infections among children less than 5 years of age and the elderly. This study intended to determine the circulating genotypes of RSV among severe acute respiratory illness (SARI) cases during the period 2016-2018 in India, among hospitalized acute febrile illness cases of age ranging from 1 to 65 years. Throat/nasopharyngeal swab samples were subjected for testing RSV and subgroups by real-time reverse transcriptase polymerase chain reaction (RT-PCR), further sequencing and phylogenetic analysis were performed for the second hypervariable region of the G gene. RSV-A and B subtypes co-circulated during the years 2016, 2017, and 2018, with RSV-A as the dominant subtype in 2016, and RSV-B as the dominant subgroup in 2017 and 2018. Phylogenetic analysis revealed that the circulating genotypes of RSV were GA2 (16/16), of RSV-A, and GB5 (23/23) of RSV-B in the South, North, and Northeast region of India during the period between 2016 and 2018. Here we report the first study comprising the distribution of RSV-A and B genotypes in the different geographic regions of India among children and adults during the year 2016 to 2018. We also report GA2.3.7 lineage of GA2 genotype for the first time in India to the best of our knowledge.
\end{abstract}

Keywords Respiratory syncytial virus $\cdot \mathrm{G}$ gene $\cdot$ Human orthopneumovirus $\cdot$ Amino acid substitution $\cdot$ Molecular epidemiology $\cdot$ India

\section{Introduction}

Acute lower respiratory tract infections are the major cause of hospitalization and death among children less than 5 years of age, with RSV being an important viral pathogen causing the infection worldwide [1,2]. Previous studies estimate that annually about 33.1 million RSV infections occur, among which about 3.2 million are hospitalized cases with around 59,600 deaths occurring in children younger than 5 years [3]. Almost every child by the age of 2 years gets infected with RSV infection, and primary infection is rarely found to be asymptomatic [4]. Reinfections are common among

Edited by Hartmut Hengel.

Arunkumar Govindakarnavar

arunviro@gmail.com; virology@manipal.edu

1 Manipal Institute of Virology (MIV), Manipal Academy of Higher Education (MAHE), Manipal, Karnataka 576104, India

2 Present Address: Vaikathu, Athrady, Udupi, Karnataka 576107, India individuals of any age group, RSV infections even lead to morbidity and mortality among the elderly [5]. The incubation period of RSV in human infection ranges from 3 to 5 days [6]. The common symptoms of RSV are nasal congestion, coryza, nonproductive cough, and mild grade fever that may lead to the involvement of the lower respiratory tract causing bronchiolitis, pneumonia, and tracheobronchitis [7-9]. The attributable mortality rate estimated due to RSV infections is around 0.86 per 1000 live births [10]. RSV infections were usually observed from March to October months in Southern Hemisphere, whereas during September to May months in Northern Hemisphere [11]. In India, RSV infections are observed during monsoons, which peaks during September and October months [12-15].

RSV genome size is approximately 15,222 nucleotides that comprise 10 genes encoding 11 proteins [16]. Based on the -antibody cross-reactivity patterns of the G glycoprotein [17], RSV has been classified into A and B subgroups that were further classified into 20 RSV-A genotypes: (GA1-7, SAA1-2, NA1-4, ON1-2, LBA1-2, CB-A, and TN1-2) and 39 RSV-B genotypes: (GB1-13, THB, BA1-14, BA-LY, BA-C, CB1, BA-CCA, BA-CCB, SAB1-4, and URU1-2) 
respectively [17-20]. Recently the RSV genotypes were reclassified for both the RSV-A and RSV-B subgroups. The reclassification of the RSV-A subgroup reported 3 genotypes (GA1-3) along with subgenotypes and lineages, whereas the reclassification of the RSV-B subgroup reported 7 genotypes (GB1-7) along with subgenotypes and lineages [21]. The current circulating genotypes among group A are GA2 and GA3 genotypes, and GB5 and GB7 genotypes among group B, worldwide [21]. In India, GA2.3.0, GA2, GA3.0.3, GA2.3.3, GA2.3.1, and GA2.3.5 genotypes for subgroup A, and GB2, GB5.0.2, GB5.0.5c, and GB5.0.2 genotypes for subgroup $B$ have been reported [14, 22, 23].

RSV can become a vaccine-preventable disease if an effective vaccine is developed against it; however, there are several vaccination strategies in advanced clinical phases for protection against severe RSV disease. For developing an effective vaccine against RSV there is a need to understand the circulating genotypes. However, there is limited data available on the molecular epidemiology of RSV among children and adults in the different geographical regions of India. Here we report a study to understand the molecular epidemiology of RSV in different geographical regions of India during the April 2016-September 2018 time period.

\section{Material and methods}

\section{Study population}

A total of $151 \mathrm{RSV}$-positive archived samples have been included in this study and cases from 10 states of India; Karnataka, Kerala, Assam, Goa, Gujarat, Maharashtra, Jharkhand, Tripura, Tamil Nadu and Odisha depicted in (Fig. 1) under Acute Febrile Illness (AFI) surveillance study conducted by Manipal Institute of Virology (MIV), with a case definition of patients admitted to hospital with fever $\left(\geq 38^{\circ} \mathrm{C}\right.$ ) of age between 1 and 65 years were recruited and samples were tested for various viral pathogens including RSV, bacterial and parasite agents by molecular and serological methods [24]. All the inpatients admitted in the hospitals fulfilling the inclusion criteria of the case definition were included in the surveillance study The surveillance study was conducted for a duration of 5 years from 2014 to 2019 [24]. The total number of the AFI samples that were obtained annually during the study period is as follows: 2016-12,414, 2017-20,359, 2018-4954. RSV-positive archived respiratory specimens of the AFI study were used in this study. The current study was reviewed and approved by the Institutional Ethical Committee, Manipal Academy of Higher Education (IEC No: UEC/32/2013-14, MUEC/ Renewal-08/2017). Cases were recruited after obtaining Informed consent as part of the AFI study.

\section{Real-time RT-PCR for subgrouping}

RNA was extracted from $150 \mu$ of 151 RT-PCR RSVpositive throat swab samples using QIAamp® Viral RNA mini kit (QIAGEN, Hilden, Germany) as per manufacturer's instructions. Real-time RT-PCR was performed using primers targeting the $\mathrm{N}$ gene that was published by $\mathrm{Hu}$ et al. using AgPath-ID ${ }^{\text {TM }}$ One-step RT-PCR kit (Applied Biosystems, Foster City, USA) to determine the RSV subgroups of the samples [25]. The reaction was performed in a QuantStudio $^{\mathrm{TM}} 5$ PCR system (Applied Biosystems, Foster City, USA). The cycling conditions for the PCR reaction were as follows: reverse transcription at $50{ }^{\circ} \mathrm{C}$ for $30 \mathrm{~min}$, initial denaturation at $95{ }^{\circ} \mathrm{C}$ for $15 \mathrm{~min}$, followed by 40 cycles of denaturation and extension at $95{ }^{\circ} \mathrm{C}$ for $15 \mathrm{~s}, 58{ }^{\circ} \mathrm{C}$ for $30 \mathrm{~s}$ respectively.

\section{Genotyping of RSV and sequencing}

Sixty-nine samples were then selected by a purposive sampling method based on the state (place), the year during the illness, and age of the patients for the determination of the genotypes. As the major number of cases were from two states, to remove the bias, 10 samples have been selected from each state based on the year during the illness. All the samples were included for genotyping from the states with samples that are less than 10 in number. Seminested PCR was carried out by using the primers targeting the second hypervariable region of the $\mathrm{G}$ gene that were published Parveen et al. using the AgPath-ID ${ }^{\mathrm{TM}}$ One-step RT-PCR kit (Applied Biosystems, Foster City, USA) [22]. The reactions were performed in a ProFlex ${ }^{\mathrm{TM}} 5$ PCR system (Applied Biosystems, Foster City, USA). The amplified products were purified using the GenElute ${ }^{\mathrm{TM}}$ Gel purification Kit (Sigma Aldrich, Missouri, USA) according to the manufacturer's instructions. The purified products were sequenced using the Big Dye Terminator kit v3.1 cycle sequencing kit (Applied Biosystems, Foster City, USA) using ABI 3500xl genetic analyzer (Applied Biosystems, Foster City, USA).

\section{Sequence analysis and phylogeny}

The sequences obtained consisted of partial regions of the second hypervariable region of the $\mathrm{G}$ gene including $\mathrm{N}$-terminal of $\mathrm{F}$ gene. The genome region ranging from 5419 to 5643 nucleotides in reference to the sequence Acc. No.: JX627336 of RSV-A subgroup, and the genome region ranging from 5349 to 5618 nucleotides in reference to the sequence Acc. No. KF640637 of the RSV-B subgroup was used for the construction of the phylogenetic tree and amino acid alignment. The published sequences of the second 


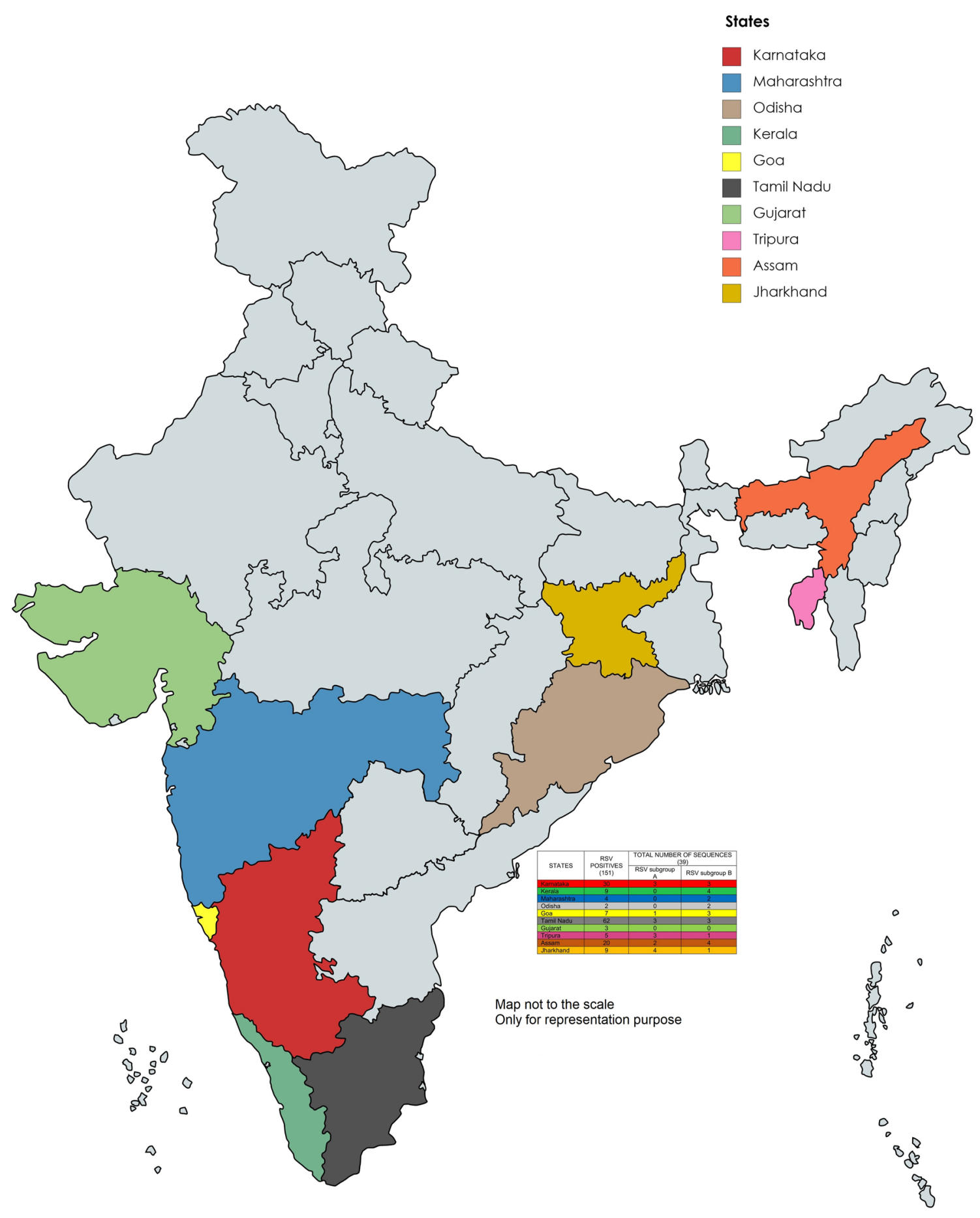

Fig. 1 Map showing ten states from which samples were represented under the AFI surveillance conducted by MIV in India. The number of sequences obtained from each state was shown in the table next to the map

hypervariable region of the G gene of RSV from different geographical regions of the world were downloaded from the GenBank of NCBI (National Centre for Biotechnology Information) database. Multiple sequence alignments of study sequences for the second hypervariable region of the G gene with known RSV genotypes that were recently classified by Goya et al. were carried out using MUSCLE (Multiple Sequence Comparison by Log Expectation) algorithms in MEGA X version 10.0.5 [21]. The phylogenetic trees of nucleotide sequences were constructed separately for RSV-A and RSV-B subgroups with Bayesian Information criterion using the Maximum Likelihood method. 
The suitable substitution model for RSV-A sequences was GTR + G substitution model and the same was used for the construction of the phylogenetic tree, whereas HKY85 + G was the suitable substitution model for RSV-B sequences and the same was used for construction of the phylogenetic tree with a support value of 1000 bootstrap replicates in PhyML software (version 3.0). The trees were visualized and midpoint trees were constructed using Figtree software. A total of 16 RSV-A and 23 RSV-B sequences of the second hypervariable region of the $\mathrm{G}$ gene were deposited in the GenBank with the accession number MN463622 to MN463637 and MN463638 to MN463660 respectively.

\section{P-distance analysis}

The intergenotypic and intragenotypic p-distances of the second hypervariable region of $\mathrm{G}$ gene sequences of RSV-A and RSV-B subgroups were calculated separately for each of the subgroups. The p-distance matrices were generated using MEGA X version 10.0.5 software.

\section{Data analysis}

Sociodemographic factors, clinical features, and laboratory parameters of the RSV cases were obtained from the AFI study database and analyzed using SPSS 15.0 for Windows software (SPSS ${ }^{\text {TM }}$ Inc, Chicago, IL, USA). For analysis of continuous variables, one-way anova test and analysis of categorical variables, the chi-square test was used. A $p$ value of $<0.05$ was set as the level of statistical significance.

\section{Results}

\section{Description of the study population}

Among AFI cases recruited, a total of 151 samples tested positive for RSV by RT-PCR during the period 2016 to 2018, out of 10 states, the major number of cases, 62 cases $(41.1 \%)$ were from Tamil Nadu, 30 (19.9\%) from Karnataka, and 20 (13.2\%) from Assam were represented in (Table 1). The mean age of the study population is 22.2 years. The positivity rate of the RSV annually for each year during the AFI study period is $0.6 \%(73 / 12414)$ in $2016,0.2 \%(42 / 20359)$ in 2017 , and $0.7 \%(36 / 4954)$ in 2018. Out of the study population, $79(52.3 \%)$ patients were males and 72 (47.7\%) were females. Most of the RSV infected cases were of low and middle socioeconomic status $75(49.7 \%)$ and $74(49 \%)$ respectively. The mean duration of hospital stay was observed to be 3.64 days. The frequencies of symptoms and signs among RSV cases were summarized in (Table 1). RSV cases were presented with symptoms like cough $(94 \%)$, general weakness
$(89.4 \%)$, and coryza $(78.1 \%)$. Coryza $(p=0.015)$, headache $(p<0.001)$, night sweats $(p=0.035)$ and joint pain ( $p=0.036)$ were significantly associated with the RSV infection among adults and children (Supplementary Table 1). Mean ESR and CRP levels were observed to be elevated.

\section{RSV subgrouping}

A total of 151 samples were subjected to RSV subgrouping of which 45 (29.8\%) were of RSV-A subgroup and 78 (51.7\%) were of RSV-B subgroup, remaining 28 (18.5\%) samples could not be subgrouped using real-time PCR. 45 RSV-A subgroup samples from each state were as follows: Tamil Nadu $(n=12)$, Karnataka $(n=11)$, Assam $(n=9)$, Jharkhand $(n=6)$, Tripura $(n=3)$, Goa $(n=2)$, and Gujarat $(n=1) .78 \mathrm{RSV}-\mathrm{B}$ subgroup samples from each state were as follows: Tamil Nadu $(n=38)$, Karnataka $(n=12)$, Kerala $(n=8)$, Assam $(n=5)$, Maharashtra $(n=4)$, Goa $(n=3)$, Gujarat $(n=2)$, Odisha $(n=2)$, Tripura $(n=2)$, and Jharkhand $(n=2)$. Cough $(p=0.005)$ was significantly associated with the RSV-A and RSV-B subgroup infections (Table 1). The mean hematological parameters total leucocyte count, differential neutrophil count, and differential lymphocyte count were found within the normal limit range among the patients with RSV-A and B subgroup infection, and even among the RSV infected adults and children (Table $2 \&$ Supplementary Table 2). The seasonality of RSV shows a clear circulation pattern of RSV each year between June and October. This circulation pattern can be correlated with the monsoon season in these regions. As can be seen in (Fig. 2) there is a cocirculation of RSV-A and B subgroups in all the three years 2016, 2017, and 2018, with RSV-A as the dominant subgroup in 2016, and RSV-B as the dominant subgroup in 2017 and 2018.

\section{Nucleotide sequencing}

A total of 39 sequences second hypervariable region of the $\mathrm{G}$ gene were obtained. Sequencing results reconfirmed the results of subgrouping done by real-time PCR. 16 RSV-A sequences from each state were as follows: Jharkhand $(n=4)$, Tripura $(n=3)$, Tamil Nadu $(n=3)$, Karnataka $(n=3)$, Assam $(n=2)$, and Goa $(n=1)$ were analyzed and used for the construction of the phylogenetic tree. $23 \mathrm{RSV}-\mathrm{B}$ sequences from each state were as follows: Jharkhand $(n=1)$, Tripura $(n=1)$, Tamil Nadu $(n=3)$, Karnataka $(n=3)$, Assam $(n=4)$, Goa $(n=3)$, Odisha $(n=2)$, Kerala $(n=4)$, and Maharashtra $(n=2)$ were analyzed and used for the construction of the phylogenetic tree. 
Table 1 Demographic, epidemiological, and clinical characteristics of RSV-A and RSV-B subgroups included in the study

\begin{tabular}{|c|c|c|c|c|c|c|}
\hline \multirow[t]{2}{*}{ Variables } & \multirow[t]{2}{*}{$N$} & \multirow[t]{2}{*}{ Present } & \multirow[t]{2}{*}{$\%$} & \multicolumn{2}{|c|}{$\operatorname{RSV}(n=123)$} & \multirow[t]{2}{*}{$p$ value } \\
\hline & & & & $\mathrm{A}$ & B & \\
\hline \multicolumn{7}{|l|}{ Gender } \\
\hline Male & 151 & 79 & 52.3 & 27 & 37 & - \\
\hline Female & 151 & 72 & 47.7 & 18 & 41 & - \\
\hline \multicolumn{7}{|c|}{$\begin{array}{l}\text { Socio Economic Status (Modi- } \\
\text { fied Udai Pareek scale) }\end{array}$} \\
\hline Low & 151 & 75 & 49.7 & 20 & 42 & - \\
\hline Middle & 151 & 74 & 49 & 24 & 35 & - \\
\hline High & 151 & 2 & 1.3 & 1 & 1 & - \\
\hline \multicolumn{7}{|l|}{ State } \\
\hline Assam & 151 & 20 & 13.2 & 9 & 5 & - \\
\hline Goa & 151 & 7 & 4.6 & 2 & 3 & - \\
\hline Gujarat & 151 & 3 & 2 & 1 & 2 & - \\
\hline Karnataka & 151 & 30 & 19.9 & 11 & 12 & - \\
\hline Kerala & 151 & 9 & 6 & 1 & 8 & - \\
\hline Maharashtra & 151 & 4 & 2.6 & 0 & 4 & - \\
\hline Odisha & 151 & 2 & 1.3 & 0 & 2 & - \\
\hline Tamil Nadu & 151 & 62 & 41.1 & 12 & 38 & - \\
\hline Tripura & 151 & 5 & 3.3 & 3 & 2 & - \\
\hline Jharkhand & 151 & 9 & 6 & 6 & 2 & - \\
\hline \multicolumn{7}{|l|}{ Year } \\
\hline 2016 & 151 & 73 & 48.3 & 38 & 13 & - \\
\hline 2017 & 151 & 42 & 27.8 & 2 & 36 & - \\
\hline 2018 & 151 & 36 & 23.8 & 5 & 29 & - \\
\hline \multicolumn{7}{|l|}{ Clinical symptoms } \\
\hline Cough & 151 & 142 & 94 & 38 & 77 & 0.005 \\
\hline General weakness & 151 & 135 & 89.4 & 39 & 73 & 0.522 \\
\hline Coryza & 151 & 118 & 78.1 & 33 & 62 & 0.736 \\
\hline Headache & 151 & 111 & 73.5 & 34 & 57 & 0.466 \\
\hline Myalgia & 151 & 102 & 67.5 & 30 & 52 & 0.842 \\
\hline Chills & 151 & 93 & 61.6 & 28 & 46 & 0.461 \\
\hline Night sweats & 151 & 62 & 41.1 & 23 & 27 & 0.073 \\
\hline Sore throat & 151 & 60 & 29.7 & 15 & 34 & 0.239 \\
\hline Joint pain & 151 & 61 & 40.4 & 16 & 32 & 0.477 \\
\hline Breathlessness & 151 & 23 & 15.2 & 7 & 13 & 0.848 \\
\hline Chest pain & 151 & 20 & 13.2 & 6 & 12 & 0.735 \\
\hline
\end{tabular}

Bold value indicates statistical significance $(p$ value $<0.05$ )

\section{Phylogenetic, amino acid, and p-distance analyses}

Phylogenetic analysis revealed that out of the 16 sequences of RSV-A subgroup, 14 sequences clustered with GA2.3.5 lineage of the GA2 genotype and 2 sequences clustered with GA2.3.7 lineage of GA2 genotype (Fig. 3), and the p-distance analysis revealed that the highest average intragenotypic p-distance was found for the GA1 genotype $(p$-distance $=0.062)$ and it was set as a threshold for the intergenotypic p-distance (Table 3). The lowest average intragenotypic p-distance was found for the GA3 genotype $(p$-distance $=0.044)$. The amino acid analysis in the partial region of the second hypervariable region of the $\mathrm{G}$ gene sequences revealed that all 16 study sequences had 24 amino acid duplication regions from 261 to 284 amino acid positions (Fig. 4). We found two substitutional changes at T292I and I319T in MN463629/MIV/Tr/India/2016, and a substitutional change at I319T in MN463635/MIV/ Jh/India/2016 study sequences which were of GA2.3.7 lineage of GA2 genotype when compared with RSV-A/ Lebanon/16LB22/2016 strain sequence of GA2.3.7 lineage of GA2 genotype sequence from 247 to 321 amino acid positions of $\mathrm{G}$ gene (Supplementary Table 3). The 14 study sequences that were of GA2.3.5 lineage of GA2 genotype 
Table 2 Laboratory parameters of RSV cases included in the study
Fig. 2 Seasonal distribution of the respiratory syncytial virus (RSV) subgroups in India during the period 2016-2018. Number of RSV cases were mentioned on $Y$-axis and time period mentioned on $X$-axis, the line graph represents the Respiratory Syncytial VirusA (RSV-A) subgroup and the dotted line graph represents the Respiratory Syncytial Virus-B (RSV-B) subgroup

\begin{tabular}{|c|c|c|c|c|}
\hline \multirow[t]{2}{*}{ Laboratory parameters at admission } & \multirow[t]{2}{*}{ All } & \multicolumn{2}{|l|}{ RSV } & \multirow[t]{2}{*}{$p$ value } \\
\hline & & A & $\mathrm{B}$ & \\
\hline Mean hospital stay (in days) $(n=151)$ & 3.64 & 3.60 & 3.67 & 0.853 \\
\hline Mean Age years $(n=151)$ & 22.2 & 19.4 & 23.4 & 0.498 \\
\hline Mean Hemoglobin levels gm/dl $(n=112)$ & 12.1 & 12.2 & 11.8 & 0.166 \\
\hline Mean Platelet count $\times 10^{3} / \mu \mathrm{l}(n=110)$ & 224 & 232 & 218 & 0.693 \\
\hline Mean Total Leucocyte count $/ \mathrm{mm}^{3}(n=111)$ & 6849 & 7038 & 7063 & 0.202 \\
\hline Mean Differential Neutrophil percentage $(n=105)$ & 61.3 & 61.7 & 61 & 0.972 \\
\hline Mean Differential Lymphocyte percentage $(n=105)$ & 31.6 & 30.9 & 32 & 0.926 \\
\hline Mean Differential Eosinophil percentage $(n=105)$ & 4.2 & 4.7 & 4 & 0.483 \\
\hline Mean Differential Monocyte percentage $(n=105)$ & 3.2 & 3 & 3.4 & 0.401 \\
\hline Mean Differential Basophil Percentage $(n=105)$ & 1.1 & 0 & 1 & 0.143 \\
\hline Mean Erythrocyte Sedimentation Rate $\mathrm{mm} / \mathrm{h}(n=22)$ & 31.8 & 29.2 & 30.6 & 0.836 \\
\hline Mean Urea levels mg/dl $(n=49)$ & 21.5 & 20.5 & 21.4 & 0.521 \\
\hline Mean Creatinine levels mg/dl $(n=48)$ & 0.8 & 0.8 & 0.7 & 0.107 \\
\hline Mean Total Bilirubin levels mg/dl $(n=71)$ & 0.5 & 0.6 & 0.5 & 0.713 \\
\hline Mean Direct Bilirubin levels mg/dl $(n=41)$ & 0.2 & 0.2 & 0.2 & 0.845 \\
\hline Mean Alkaline Phosphatase levels IU/L $(n=46)$ & 392.4 & 442.9 & 346.2 & 0.478 \\
\hline Mean C-Reactive protein levels mg/l $(n=69)$ & 15.6 & 14.5 & 15.8 & 0.940 \\
\hline Mean Aspartate aminotransferase levels IU/L $(n=71)$ & 46.3 & 45.7 & 47.6 & 0.925 \\
\hline Mean Alanine aminotransferase levels IU/L $(n=71)$ & 31.3 & 36.5 & 25 & 0.172 \\
\hline
\end{tabular}

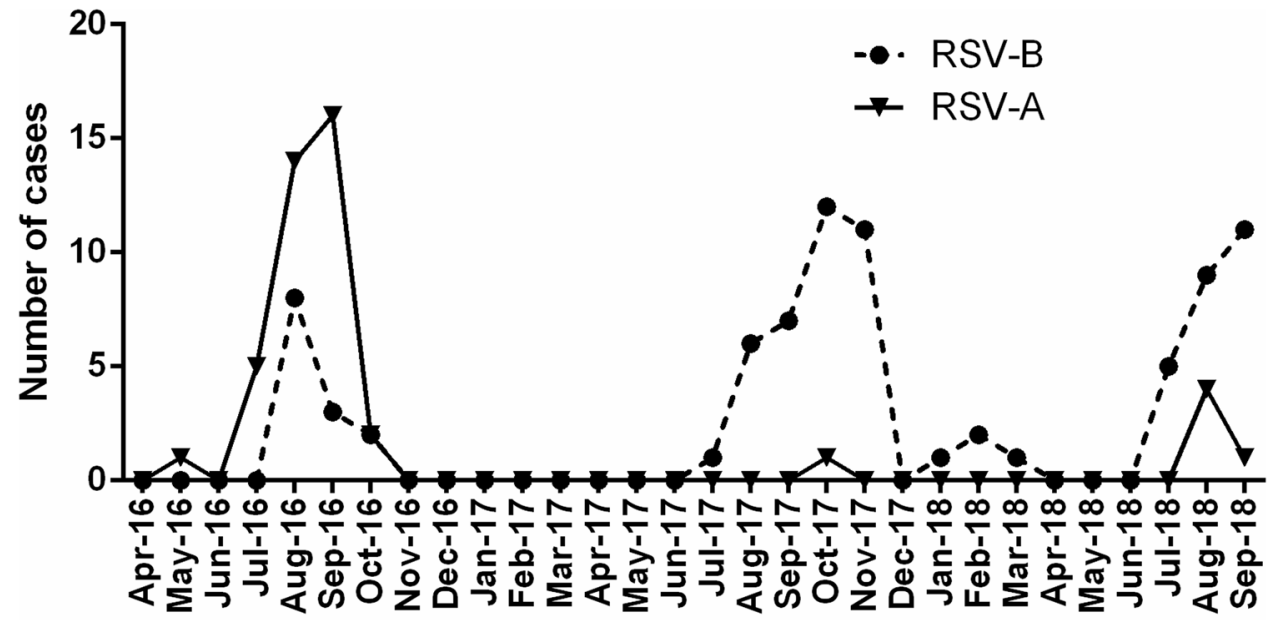

Time period in months were compared with the ON67-1210A strain sequence of GA2.3.5 lineage of GA2 genotype from 247 to 321 amino acid positions of the $\mathrm{G}$ gene. The amino acid substitutional changes were detected in 12 of these 14 sequences, whereas substitutional changes were not observed in the remaining two sequences (Supplementary Table 4). The aminoacid substitutional changes L248I ( $n=2$ sequences), G254R $(n=1), \mathrm{H} 258 \mathrm{Q}(n=1), \mathrm{E} 262 \mathrm{~K}(n=3), \mathrm{L} 265 \mathrm{~F}(n=1)$, H266Y $(n=1), \mathrm{E} 271 \mathrm{~K}(n=2), \mathrm{L} 274 \mathrm{P}(n=7), \mathrm{L} 289 \mathrm{P}$ $(n=1), \mathrm{L} 298 \mathrm{P}(n=7), \mathrm{V} 303 \mathrm{~A}(n=4), \mathrm{Y} 304 \mathrm{H}(n=6)$,
E308K $(n=1)$, L314P $(n=1)$, and T319I $(n=2)$ were observed in our study sequences. New aminoacid substitutional changes L248T ( $n=2$ sequences), T281A $(n=1)$, $\mathrm{E} 287 \mathrm{~K}(n=1), \mathrm{S} 294 \mathrm{P}(n=1), \mathrm{G} 296 \mathrm{D}(n=1), \mathrm{V} 303 \mathrm{I}(n=1)$, T319S $(n=1)$, T319N $(n=1)$, and T320A $(n=4)$ were observed in our study sequences.

Phylogenetic analysis revealed that all the 23 sequences of RSV-B subgroup clustered with GB5.0.5a lineage of the GB5 genotype (Fig. 5), the p-distance analysis was carried out for 6 genotypes (GB1, GB2, GB4, GB5, GB6, and GB7), 


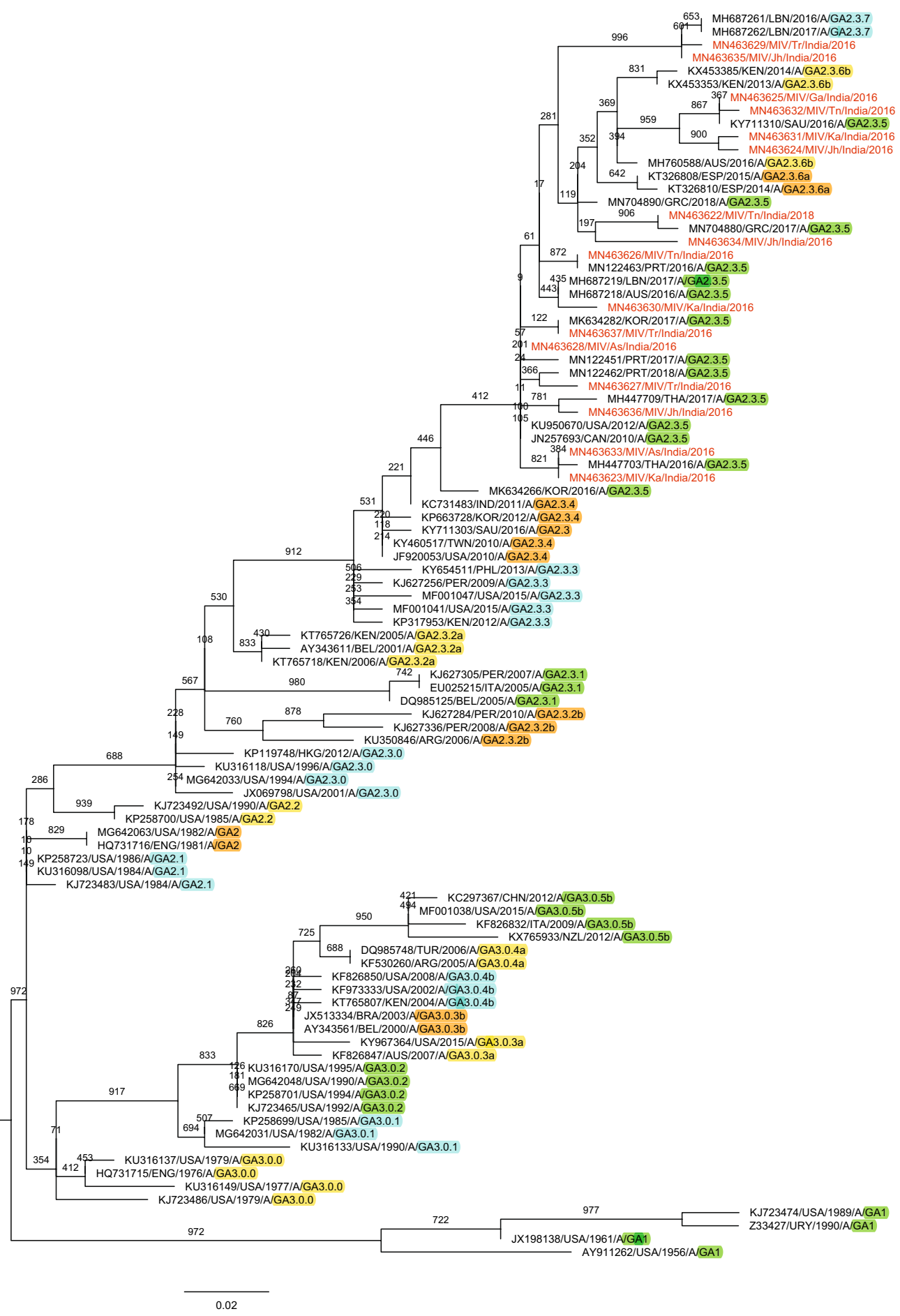

Fig. 3 Mid-rooted phylogenetic tree of RSV-A subgroup. Mid-rooted phylogenetic tree of the second hypervariable region of the RSV-A $\mathrm{G}$ protein gene constructed by using Maximum Likelihood method, by $\mathrm{GTR}+\mathrm{G}$ substitution model with 1000 bootstrap replicates in PhyML software. Study sequences are displayed in red. The reference sequences used to construct the tree were downloaded from the Gen-
Bank database. The two lettered words in the labels of the MIV isolates represent the state names, where: $\mathrm{T} n=$ Tamil Nadu, Ka=Karnataka, $\mathrm{Jh}=$ Jharkhand, $\mathrm{Ga}=\mathrm{Goa}, \mathrm{Tr}=$ Tripura, and As $=$ Assam. The grid lines over the tree represent the patristic distance where the distance between each grid is 0.005 . The names of the genotypes and lineages were highlighted by different colors 
Table 3 Average intra and intergenotypic p-distance of RSV-A subgroup

\begin{tabular}{llll}
\hline & GA1 & GA2 & GA3 \\
\hline GA1 & $\underline{\mathbf{0 . 0 6 2}}$ & & \\
GA2 & 0.166 & $\mathbf{0 . 0 5 7}$ & \\
GA3 & 0.163 & 0.115 & $\mathbf{0 . 0 4 4}$
\end{tabular}

The intragenotypic p-distances are denoted in bold. The highest intragenotypic p-distance underlined

the highest average intragenotypic p-distance was obtained for the GB1 genotype ( $p$-distance $=0.070)$ and it was set as a threshold for the intergenotypic p-distance (Table 4). The lowest average intragenotypic p-distance was found for GB4 genotype ( $p$-distance $=0.015$ ). The amino acid analysis in the partial region of the second hypervariable region of the $\mathrm{G}$ gene sequences revealed that all 23 study sequences had 20 amino acid duplication regions from 240 to 269 amino acid positions (Fig. 6). These 23 study sequences were compared with the RSV-B/England583/2013 strain sequence of GB5.0.5a lineage of GB5 genotype from 223 to 312 amino acid positions of the $\mathrm{G}$ gene. The amino acid substitutional changes were detected in 22 of these 23 sequences, whereas substitutional changes were not observed in the remaining one sequence (Supplementary Table 5). The aminoacid substitutional changes T227N ( $n=1$ sequence), I254T $(n=5)$, $\mathrm{S} 267 \mathrm{P}(n=3), \mathrm{S} 269 \mathrm{~F}(n=3), \mathrm{I} 270 \mathrm{~T}(n=4), \mathrm{A} 271 \mathrm{~V}(n=3)$, D273N $(n=1)$, Y287H $(n=3)$, T290I $(n=8)$, and T312I $(n=8)$ were reported in our study [18, 26-28]. New aminoacid substitutional changes P231L ( $n=6$ sequences), P235L $(n=2), \mathrm{E} 241 \mathrm{~K}(n=1), \mathrm{D} 243 \mathrm{~V}(n=1), \mathrm{P} 247 \mathrm{~L}$ $(n=2), \mathrm{H} 259 \mathrm{Y}(n=1), \mathrm{E} 261 \mathrm{G}(n=2), \mathrm{K} 278 \mathrm{R}(n=5)$, S285L ( $n=1$ from Tripura), L286P $(n=1), \mathrm{A} 303 \mathrm{P}(n=1)$, and $\mathrm{S} 311 \mathrm{~F}(n=1)$ were observed in our study sequences. The old genotypes which were corresponding to respective newly reclassified genotypes are depicted in (Supplementary Table 6).

\section{Discussion}

RSV is one of the most common viral causes of acute respiratory illness (ARI) among infants and young children throughout the world [23]. In India, most of the molecular epidemiological studies were conducted in Assam, New Delhi, and Maharashtra to analyze the circulation and genetic diversity of RSV [5, 22, 23, 29, 30]. This study is the first of its kind which has covered 10 states, Karnataka, Kerala, Tamil Nadu, Assam, Goa, Gujarat, Maharashtra, Jharkhand, Tripura, and Odisha, and even different geographical locations that lie in southern, eastern, and western parts of India were analyzed from April 2016 to September 2018. Most of the molecular epidemiological studies of RSV usually focus only on children less than 5 years of age [1, 31]. In this study, we included cases from the age group 1-65 years. In the current study, phylogenetic analyses were performed based on the newly reclassified genotypes by Goya et al. [21]. Phylogenetic analysis of RSV-A subgroup revealed a novel genetic lineage GA2.3.7 for the first time in India to the best of our knowledge, including GA2.3.5 lineage of GA2 genotype. The distinct clade of GA2.3.7 lineage of GA2 genotype showed the patristic distance of approximately 0.120 when the distance was set as 0 (zero) at the ancestral node of 2.3.0 subgenotype, fulfilling the criteria to designate as a new genetic lineage having more than eight multiples of the patristic distance of 0.015 which was proposed by Goya et al. [21]. The patristic distance of 2.3.0 subgenotypes and further lineages in it is depicted in (Supplementary Fig. 1). GA2.3.7 lineage of GA2 genotype was first reported in 2016 in Lebanon with 72 bp nucleotide duplication similar to GA2.3.5 lineage of GA2 genotype with characteristic amino acid substitutions (G284S, E295K, Y304H, L314P, T319I, and P320K) [18]. In the current study sequences of GA2.3.7 lineage, a substitutional change I319T was observed in both the GA2.3.7 lineage study sequences, whereas T292I was observed in a sequence from

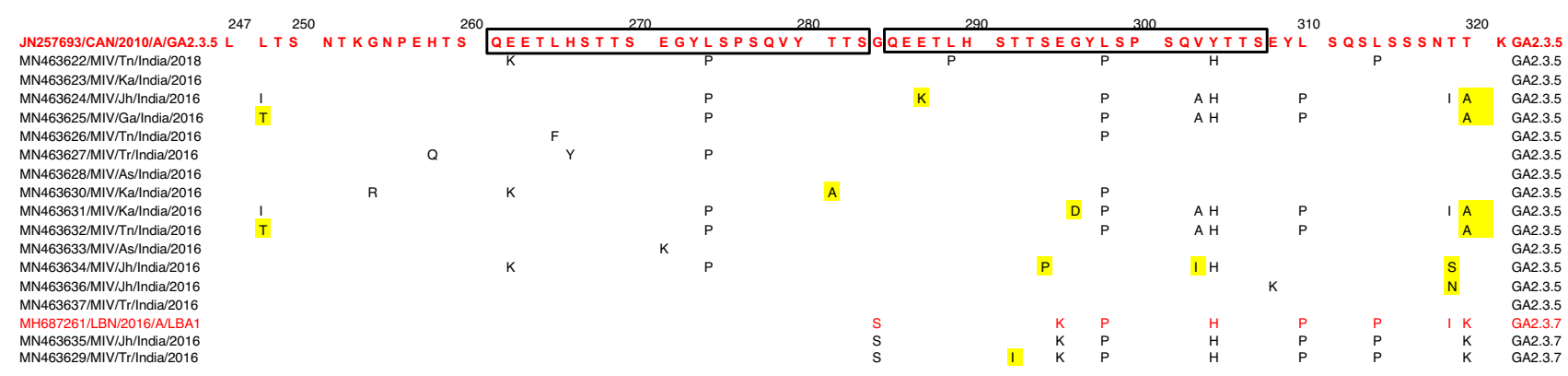

Fig. 4 Multiple amino acid alignments of partial region of the second hypervariable region of $\mathrm{G}$ protein gene RSV-A subgroup sequences. Alignments are shown relative to the ON67-1210A sequence strain of NA1 genotype, aminoacid positions (247-321). Dots represent iden-

tical residues. Study sequences are in black font, whereas reference sequences in red font. Newly identified substitutions highlighted yellow in color 
Tripura when compared to RSV-A/Lebanon/16LB22/2016 strain sequence of GA2.3.7 lineage which was not observed in any of the GA2.3.7 lineage sequences that were reported by Abou-El-Hassan et al. [18]. We observed the circulation of GA2.3.7 lineage of GA2 genotype in 2016 among children in our study. The GA2.3.5 lineage of GA2 genotype RSV-A was first detected in Canada in 2011 and became the predominant genotype globally [32]. In the current study, we observed GA2.3.5 lineage of GA2 genotype as the predominant circulating genotype of RSV-A subgroup in the South, North, and Northeast regions of India during 2016-2018. Sahu et al. (Madhya Pradesh) reported the substitutional changes E262K, L274P, L298P, V303A, and L310P; Haider et al. (Delhi) reported the substitutional changes E262K, L274P, and L298P; Choudhary et al. (Maharashtra) reported the substitutional changes L274P, L298P, V303A, and L310P which were even observed in our study sequences $[5,23,30]$. The amino acid substitutional changes that were mentioned earlier in the results section (i.e., from L248I to T320A) were also reported by Cui et al., Ogunsemowo et al., Malasao et al., and Eshaghi et al. [32-35]. Few of the newly identified substitutional changes were also observed in our study sequences, but these changes did not constitute for defining these sequences as novel genotype based on the classification criteria defined by Goya et al. The circulating genotypes from different countries during the same period of our study (i.e., 2016, 2017, and 2018) were replicated same as our findings as GA2.3.5 lineage of GA2 genotype in South Korea, Portugal, Greece, Australia, Saudi Arabia, Lebanon, and Thailand [18, 26-28, 36-38]. In India, according to old classification previous studies reported circulation of GA2, GA5 genotypes (2001-2005), and ON1 genotype (2011-2015) in Delhi; GA5 and NA1 genotypes (2009-2012) in Assam; and NA1 lineage and ON1 genotypes in Pune (2009-2012), and NA1 and ON1 genotypes in Kerala (2012-2014) [14, 22, 23, 30, 39, 40]. At the time of preparing this manuscript, a publication by Broor et al. in 2019 related to the status of the molecular epidemiology of RSV in India was the latest information related to the circulated genotypes in India to the best of our knowledge [41].

Phylogenetic analysis of the RSV-B subgroup revealed GB5.0.5a lineage of GB5 genotype as the predominant circulating genotype in the South, North, and Northeast regions of India. GB5.0.5a was first detected in 2013 from England (Accession number: KY249660) from unpublished data with $60 \mathrm{bp}$ nucleotide duplication region along with twelve signature amino acids [21]. Sahu et al. (Madhya Pradesh) reported the substitutional changes I254T, E261G, S267P, I270T, and Y287H; Haider et al. (Delhi) reported the substitutional changes T227N, E241K, I254T, S267P, I270T, A271V, Y287H, and T290I; Choudhary et al. (Maharashtra) reported the substitutional changes T227N, E261G, S267P, I270T, A271V, Y287H, and T290I which were even observed in our study sequences [5, 23, 42]. The amino acid substitutional changes that were mentioned earlier in the results section (i.e., from T227N to T312I) were also reported by Abou-El-Hassan et al., Tsergouli et al., Yun et al., and Al-Hassinah et al. [18, 26-28]. The circulating genotypes from different countries during the same period of our study (i.e., 2016, 2017, and 2018) were found to be of GB5.0.5a lineage of GB5 genotype in South Korea, Portugal, Greece, Australia, Saudi Arabia, Lebanon, and Thailand [18, 26-28, 36-38]. In India, according to the old classification, previous studies reported circulation of BA genotype (2001-2005), BA7, BA9, BA10, BA12 genotypes (2007-2010), SAB4, BA8, and BA9 genotypes (2011-2015) in Delhi; BA genotype (2005 -2008) in Kolkata; GB2, BA9, and BA12 genotypes (2009-2012) in Maharashtra; BA9 and BA10 genotypes (2012-2014) in Kerala [14, 22, 23, 39, 40, 42]. The p-distance analysis was carried out for 6 genotypes (GB1, GB2, GB4, GB5, GB6, and GB7) where 1 genotype (GB3) was excluded from the analysis as it had lacked signature amino acids significant for the genotypic determination in the second hypervariable region of the G gene [21].

Co-circulation of A and B subgroups were observed in this study, in 2016 with a predominance of RSV-A subgroup, whereas the RSV-B subgroup was predominant in 2017 and 2018. Similar studies with co-circulation of RSV-A and RSV-B were observed with RSV-B as a predominant subgroup in 2005-2006, whereas RSV-A in 2007-2008 in Kolkata [14]. RSV-B was observed to be predominant among co-circulating subgroups (2001-2002), RSV-A was observed to be predominant during (2002-2003, 2003-2004, and 2004-2005) in Delhi [22]. A study that analyzed the laboratory parameters of RSV infected individuals reported that the mean values of WBC count, ESR levels, and CRP levels as 9840 cells/ $\mathrm{mm}^{3}, 28 \mathrm{~mm} / \mathrm{h}$, and $27 \mathrm{mg} / \mathrm{l}$, respectively [43]. In this study, we found the mean values of WBC count, ESR levels, and CRP levels were as follows: 6849 cells $/ \mathrm{mm}^{3}$, $31.8 \mathrm{~mm} / \mathrm{h}$, and $15.6 \mathrm{mg} / \mathrm{l}$ respectively.

The circulation and spread of the GA2 genotype, and the GB5 genotype in India might be due to the change of the viral antigenic properties leading to the emergence of the new genotypes for evasion from the host immune response. The disappearance of old genotypes with the replacement of new genotypes of RSV might be because of the herd immunity developed by the individuals [44]. The development of a vaccine against circulating genotypes in a particular region can reduce the extent of the spread of RSV infection among children and old age.

This study has some limitations. Sequences of few samples were unable to obtain as the quality of the RNA was low as samples were from retrospective study. The newly identified substitutional changes need to be seen in further studies 


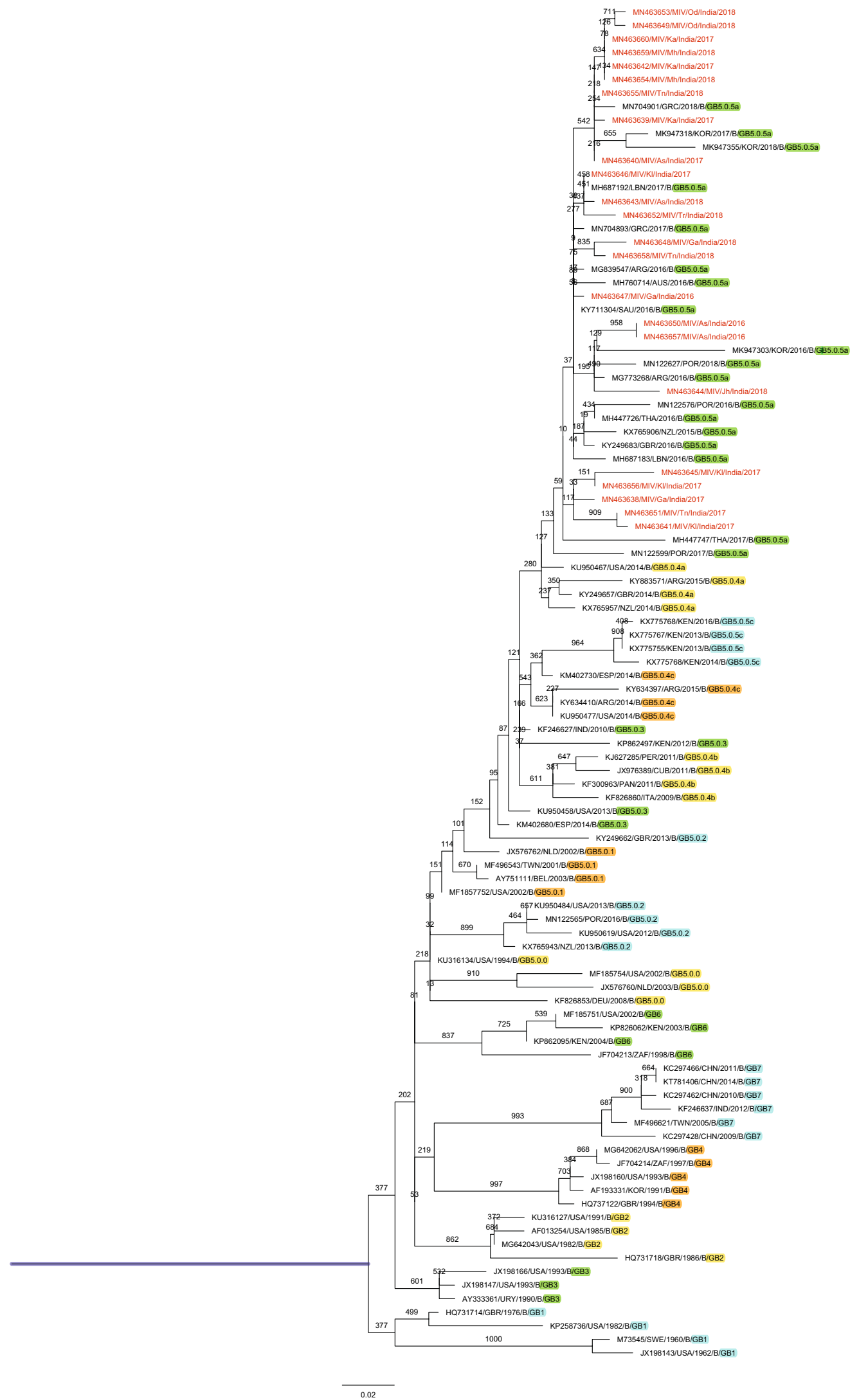


4Fig. 5 Mid-rooted phylogenetic tree of RSV-B subgroup. Mid-rooted phylogenetic tree of the second hypervariable region of the RSV-B $\mathrm{G}$ protein gene constructed by using Maximum Likelihood method, by $\mathrm{HKY} 85+\mathrm{G}$ substitution model with 1000 bootstrap replicates in PhyML software. Study sequences are displayed in red. The reference sequences used to construct the tree were downloaded from the GenBank database. The two lettered words in the labels of the MIV isolates represent the state names, where: T $n=$ Tamil Nadu, $\mathrm{Ka}=$ Karnataka, $\mathrm{Jh}=\mathrm{Jharkhand}, \mathrm{Ga}=\mathrm{Goa}, \mathrm{Tr}=$ Tripura, $\mathrm{As}=$ Assam, $\mathrm{Kl}=$ Kerala, $\mathrm{Od}=$ Odisha, and $\mathrm{Mh}=$ Maharashtra The grid lines over the tree represent the patristic distance where the distance between each grid is 0.005 . The names of the genotypes and lineages were highlighted by different colors regions of India during the period between 2016 and 2018 . To the best of our knowledge, this is the first report to identify the GA2.3.7 lineage of GA2 genotype in India. RSV needs more surveillance studies on molecular epidemiology to get a better understanding of the circulating genotypes globally as we are observing mutations. Acute surveillance is required to know the predominance of RSV-A and RSV-B subgroups which may help in the implementation of the development of therapeutics or prevention against RSV infection. The consequences of the
Table 4 Average intra and intergenotypic p-distance of RSV-B subgroup

\begin{tabular}{lllllll}
\hline & GB1 & GB2 & GB4 & GB5 & GB6 & GB7 \\
\hline GB1 & $\underline{\mathbf{0 . 0 7 0}}$ & & & & & \\
GB2 & 0.091 & $\mathbf{0 . 0 2 8}$ & & & & \\
GB4 & 0.114 & 0.086 & $\mathbf{0 . 0 1 5}$ & & & \\
GB5 & 0.100 & 0.074 & 0.103 & $\mathbf{0 . 0 4 5}$ & & \\
GB6 & 0.102 & 0.074 & 0.094 & 0.076 & $\mathbf{0 . 0 3 6}$ & \\
GB7 & 0.103 & 0.081 & 0.108 & 0.099 & 0.103 & $\mathbf{0 . 0 2 0}$ \\
\hline
\end{tabular}

The intragenotypic p-distances are denoted in bold. The highest intragenotypic p-distance underlined

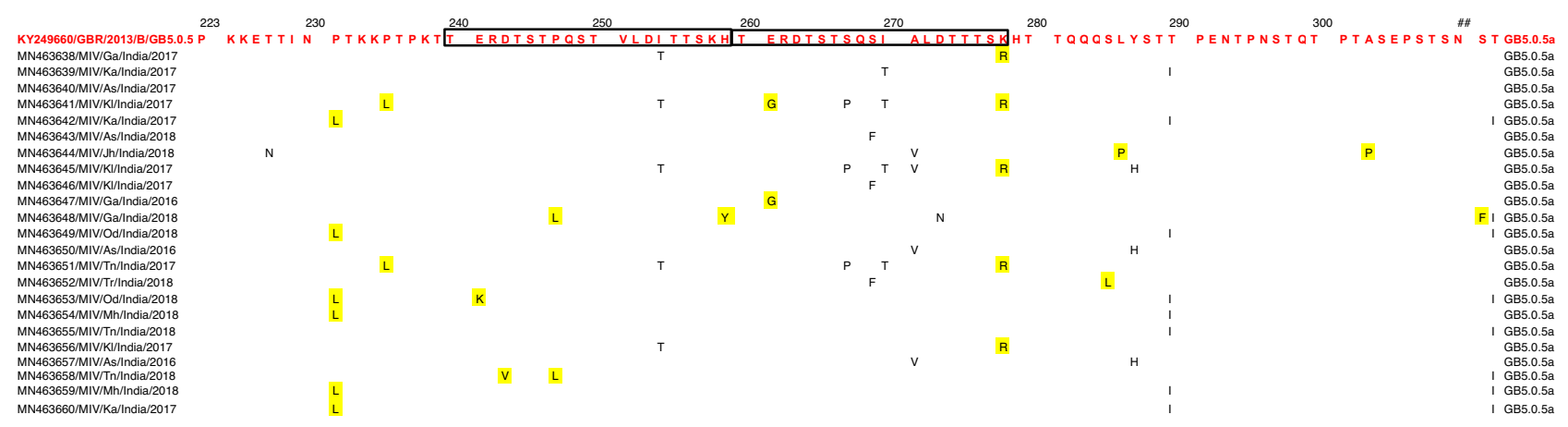

Fig. 6 Multiple amino acid alignments of partial region of the second hypervariable region of $\mathrm{G}$ protein gene RSV-B subgroup sequences. Alignments are shown relative to the RSV-B/England583/2013 sequence strain of GB5.0.5a genotype, aminoacid positions (223-

in other parts of India. The p-distance analysis was carried out for 6 genotypes (GB1, GB2, GB4, GB5, GB6, and GB7) where 1 genotype (GB3) was excluded from the analysis as it doesn't have characteristic amino acid significant for the genotypic determination in the second hypervariable region of the $\mathrm{G}$ gene.

\section{Conclusions}

GA2 genotype was the circulating genotype of the RSV-A subgroup, whereas GB5 was the circulating genotype of the RSV-B subgroup in the South, North, and Northeast
312). Dots represent identical residues. Study sequences are in black font, whereas reference sequence in red font. Newly identified substitutions highlighted yellow in color

COVID19 pandemic and the restrictions applied to the movement of the population will be the most useful aspect leading to the decrease of the transfer of RSV infection among the population worldwide. If there is a possibility in maintaining the same social distancing norms even after the fall of COVID19 can lead to a massive decline in the infection rate of RSV and other transmissible diseases.

Supplementary Information The online version contains supplementary material available at https://doi.org/10.1007/s11262-021-01859-4.

Acknowledgements We acknowledge our sincere gratitude to Mr. Sanjay Ramachandran, Research Officer, and all the laboratory technicians of the Manipal Institute of Virology, Manipal, Karnataka, India. 
Author contributions G.A conceptualization, methodology, supervision, funding, resources acquisition, writing - review \& editing. B.S.S investigation, analysis, writing-original draft. S.D methodology supervision, writing — review \& editing, formal analysis. R.B formal analysis, writing-review \& editing, data curation. O.C.D formal analysis, writing—review \& editing.

Funding Open access funding provided by Manipal Academy of Higher Education, Manipal. This work was supported by "Indian Council of Medical Research, Department of Health and Family Welfare, Government of India" for the Virus Research and Diagnostics Laboratories (File no.5/8/7/15/2010/ECD-I) to Govindakarnavar Arunkumar.

Data availability Supporting data was made available in supplementary material 1 which can be made available for the reader.

\section{Declarations}

Conflict of interest All authors: no reported conflicts of interest. All authors have submitted the ICMJE form for disclosure of potential conflicts of interest. Conflicts that the editors consider relevant to the content of the manuscript have been disclosed.

Consent for publication All authors have seen and approved the manuscript.

Ethical approval The current study was reviewed and approved by the Institutional Ethical Committee, Manipal Academy of Higher Education (IEC No: UEC/32/2013-14, MUEC/Renewal-08/2017). Cases were recruited after obtaining Informed consent as part of the AFI study.

Open Access This article is licensed under a Creative Commons Attribution 4.0 International License, which permits use, sharing, adaptation, distribution and reproduction in any medium or format, as long as you give appropriate credit to the original author(s) and the source, provide a link to the Creative Commons licence, and indicate if changes were made. The images or other third party material in this article are included in the article's Creative Commons licence, unless indicated otherwise in a credit line to the material. If material is not included in the article's Creative Commons licence and your intended use is not permitted by statutory regulation or exceeds the permitted use, you will need to obtain permission directly from the copyright holder. To view a copy of this licence, visit http://creativecommons. org/licenses/by/4.0/.

\section{References}

1. Etemadi MR, Sekawi Z, Othman N, Lye M-S, Moghaddam FY (2013) Circulation of human respiratory syncytial virus strains among hospitalized children with acute lower respiratory infection in Malaysia. Evol Bioinform. https://doi.org/10.4137/EBO. S10999

2. Forum of International Respiratory Societies (2017) The global impact of respiratory disease, 2nd edn. European Respiratory Society, Sheffield

3. Shi T (2017) Global, regional, and national disease burden estimates of acute lower respiratory infections due to respiratory syncytial virus in young children in 2015: a systematic review and modelling study. Lancet 390:13

4. Popow-Kraupp T, Aberle JH (2011) Diagnosis of respiratory syncytial virus infection. Open Microbiol J 30(5):128-134
5. Sahu M, Shukla MK, Barde PV (2017) Molecular characterization of human respiratory syncytial virus detected from central India. J Med Virol 89(10):1871-1874

6. Peter LC, Ruth AK (2013) Respiratory syncytial virus and metapneumovirus. In: Knipe DM, Howley PM, Cohen JI, Griffin DE, Lamb RA, Martin MA, Racaniello VR, Roizman B (eds) Fields virology, 6th edn. Lippincott Williams \& Wilkins a Wolters Kluwer Business, Philadelphia, pp 1086-1123

7. Simoes EAF (1999) Respiratory syncytial virus infection. Lancet 354:6

8. Falsey AR, Walsh EE (2000) Respiratory syncytial virus infection in adults. Clin Microbiol Rev 13:14

9. Glezen WP (1986) Risk of primary infection and reinfection with respiratory syncytial virus. Arch Pediatr Adolesc Med 140(6):543

10. Geoghegan S, Erviti A, Caballero MT, Vallone F, Zanone SM, Losada JV et al (2017) Mortality due to respiratory syncytial virus. Burden and risk factors. Am J Respir Crit Care Med 195(1):96-103

11. Obando-Pacheco P, Justicia-Grande AJ, Rivero-Calle I, Rodríguez-Tenreiro C, Sly P, Ramilo O et al (2018) Respiratory syncytial virus seasonality: a global overview. J Infect Dis 217(9):1356-1364

12. Yeolekar LR, Damle RG, Kamat AN, Khude MR, Simha V, Pandit AN (2008) Respiratory viruses in acute respiratory tract infections in Western India. Indian J Pediatr 75(4):341-345

13. Bharaj P, Sullender WM, Kabra SK, Mani K, Cherian J, Tyagi V et al (2009) Respiratory viral infections detected by multiplex PCR among pediatric patients with lower respiratory tract infections seen at an urban hospital in Delhi from 2005 to 2007. Virol J 26(6):89

14. Agrawal AS, Sarkar M, Ghosh S, Chawla-Sarkar M, Chakraborty N, Basak M et al (2009) Prevalence of respiratory syncytial virus group B genotype BA-IV strains among children with acute respiratory tract infection in Kolkata, Eastern India. J Clin Virol 45(4):358-361

15. Broor S, Parveen S, Bharaj P, Prasad VS, Srinivasulu KN, Sumanth KM et al (2007) A prospective three-year cohort study of the epidemiology and virology of acute respiratory infections of children in rural India. PLoS ONE 2(6):e491

16. Rima B, Collins P, Easton A, Fouchier R, Kurath G, Lamb RA et al (2017) ICTV virus taxonomy profile: pneumoviridae. J Gen Virol 98(12):2912-2913

17. Malekshahi SS, Razaghipour S, Samieipoor Y, Hashemi FB, Manesh AAR, Izadi A et al (2019) Molecular characterization of the glycoprotein and fusion protein in human respiratory syncytial virus subgroup a: emergence of ON-1 genotype in Iran. Infect Genet Evol 71:166-178

18. Abou-El-Hassan H, Massaad E, Soudani N, Assaf-Casals A, Shaker R, Lteif Khoury M, Ghanem S, Karam M, Andary R, Saito R, Ghassan D, Zaraket H (2019) Detection of ON1 and novel genotypes of human respiratory syncytial virus and emergence of palivizumab resistance in Lebanon. PLoS ONE 14(2):e0212687

19. Cui G, Zhu R, Qian Y, Deng J, Zhao L, Sun Y et al (2013) genetic variation in attachment glycoprotein genes of human respiratory syncytial virus subgroups A and B in children in recent five consecutive years. PLoS ONE 8(9):e75020

20. Gaymard A, Bouscambert-Duchamp M, Pichon M, Frobert E, Vallee J, Lina B et al (2018) Genetic characterization of respiratory syncytial virus highlights a new BA genotype and emergence of the ON1 genotype in Lyon, France, between 2010 and 2014. J Clin Virol 102:12-18

21. Goya S, Galiano M, Nauwelaers I, Trento A, Openshaw PJ, Mistchenko AS et al (2020) Toward unified molecular surveillance of RSV: a proposal for genotype definition. Influenza Other Respir Viruses 14(3):274-285 
22. Parveen S, Sullender WM, Fowler K, Lefkowitz EJ, Kapoor SK, Broor S (2006) Genetic variability in the G protein gene of group A and B respiratory syncytial viruses from India. J Clin Microbiol 44(9):3055-3064

23. Choudhary ML, Anand SP, Wadhwa BS, Chadha MS (2013) Genetic variability of human respiratory syncytial virus in Pune, Western India. Infect Genet Evol 20:369-377

24. Arunkumar G (2017) Annual report of Hospital based surveillance of Acute Febrile Illness in India. Manipal Centre for Virus Research. https://manipal.edu/content/dam/manipal/mu/ dovr/Documents/AFI/MCVR_AFI\%20Brochure\%20final.pdf. Accessed 21 Aug 2019

25. Hu A, Colella M, Tam JS, Rappaport R, Cheng S-M (2003) Simultaneous detection, subgrouping, and quantitation of respiratory syncytial virus A and B by real-time PCR. J Clin Microbiol 41(1):149-154

26. Al-Hassinah S, Parveen S, Somily AM, AlSaadi MM, Alamery SF, Haq SH et al (2020) Evolutionary analysis of the ON1 genotype of subtype a respiratory syncytial virus in Riyadh during 2008-16. Infect Genet Evol 79:104153

27. Yun KW, Choi EH, Lee HJ (2020) Molecular epidemiology of respiratory syncytial virus for 28 consecutive seasons (19902018 ) and genetic variability of the duplication region in the $\mathrm{G}$ gene of genotypes ON1 and BA in South Korea. Arch Virol 165(5):1069-1077

28. Tsergouli K, Pappa S, Haidopoulou K, Gogou M, Giannopoulos A, Papa A (2019) Respiratory syncytial virus in greece, 2016-2018. Intervirology 62(5-6):210-215

29. Biswas D, Yadav K, Borkakoty B, Mahanta J (2013) Molecular characterization of human respiratory syncytial virus NA1 and GA5 genotypes detected in Assam in northeast India, 20092012: RSV Genotypes in Dibrugarh (Assam), Northeast India. J Med Virol 85(9):1639-1644

30. Haider MSH, Deeba F, Khan WH, Naqvi IH, Ali S, Ahmed A et al (2018) Global distribution of NA1 genotype of respiratory syncytial virus and its evolutionary dynamics assessed from the past 11 years. Infect Genet Evol 60:140-150

31. Park E, Park PH, Huh JW, Yun HJ, Lee HK, Yoon MH et al (2017) Molecular and clinical characterization of human respiratory syncytial virus in South Korea between 2009 and 2014. Epidemiol Infect 145(15):3226-3242

32. Eshaghi A, Duvvuri VR, Lai R, Nadarajah JT, Li A, Patel SN et al (2012) Genetic variability of human respiratory syncytial virus a strains circulating in Ontario: a novel genotype with a 72 nucleotide G gene duplication. PLoS ONE 7(3):e32807

33. Cui G, Qian Y, Zhu R, Deng J, Zhao L, Sun Y et al (2013) Emerging human respiratory syncytial virus genotype ON1 found in infants with pneumonia in Beijing, China. Emerg Microbes Infect 2(4):e22
34. Ogunsemowo O, Olaleye DO, Odaibo GN (2018) Genetic diversity of human respiratory syncytial virus circulating among children in Ibadan, Nigeria. PLoS ONE 13(1):e0191494

35. Malasao R, Okamoto M, Chaimongkol N, Imamura T, Tohma K, Dapat I et al (2015) Molecular characterization of human respiratory syncytial virus in the philippines, 2012-2013. PLoS ONE 10(11):e0142192

36. Sáez-López E, Cristóvão P, Costa I, Pechirra P, Conde P, Guiomar R et al (2019) Epidemiology and genetic variability of respiratory syncytial virus in Portugal, 2014-2018. J Clin Virol 121:104200

37. Di Giallonardo F, Kok J, Fernandez M, Carter I, Geoghegan J, Dwyer D et al (2018) Evolution of human respiratory syncytial virus (RSV) over multiple seasons in New South Wales, Australia. Viruses 10(9):476

38. Thongpan I, Suntronwong N, Vichaiwattana P, Wanlapakorn N, Vongpunsawad S, Poovorawan Y (2019) Respiratory syncytial virus, human metapneumovirus, and influenza virus infection in Bangkok, 2016-2017. Peer J 7:e6748

39. Raghuram SV, Khan WH, Deeba F, Sullender W, Broor S, Parveen S (2015) Retrospective phylogenetic analysis of circulating BA genotype of human respiratory syncytial virus with 60 bp duplication from New Delhi, India during 2007-2010. VirusDisease 26(4):276-281

40. Patil SL, Balakrishnan A (2017) Genetic characterization respiratory syncytial virus in Kerala, the southern part of India. J Med Virol 89(12):2092-2097

41. Broor S, Parveen S, Maheshwari M (2018) Respiratory syncytial virus infections in India: epidemiology and need for vaccine. Indian J Med Microbiol 36(4):458

42. Haider MSH, Khan WH, Deeba F, Ali S, Ahmed A, Naqvi IH et al (2018) BA9 lineage of respiratory syncytial virus from across the globe and its evolutionary dynamics. PLOS ONE 13(4):e0193525

43. Saijo M, Ishii T, Kokubo M, Murono K, Takimoto M, Fujita K (1996) White blood cell count, C-reactive protein and erythrocyte sedimentation rate in respiratory syncytial virus infection of the lower respiratory tract. Pediatr Int 38(6):596-600

44. Choi EH, Lee HJ (2000) Genetic diversity and molecular epidemiology of the $G$ protein of subgroups A and B of respiratory syncytial viruses isolated over 9 consecutive epidemics in Korea. J Infect Dis 181(5):1547-1556

Publisher's Note Springer Nature remains neutral with regard to jurisdictional claims in published maps and institutional affiliations. 